\title{
A power efficient MAC protocol for wireless body area networks
}

\author{
Moshaddique Al Ameen, Niamat Ullah, M Sanaullah Chowdhury, SM Riazul Islam and Kyungsup Kwak*
}

\begin{abstract}
Applications of wearable and implanted wireless sensor devices are hot research area. A specialized field called the body area networks (BAN) has emerged to support this area. Managing and controlling such a network is a challenging task. An efficient media access control (MAC) protocol to handle proper management of media access can considerably improve the performance of such a network. Power consumption and delay are major concerns for MAC protocols in a BAN. Low cost wakeup radio module attached with sensor devices can help reduce power consumption and prolong the network lifetime by reducing idle state power consumption and increasing sleep time of a BAN node. In this article, we propose a new MAC protocol for BAN using out of band (on-demand) wakeup radio through a centralized and coordinated external wakeup mechanism. We have compared our method against some existing MAC protocols. Our method is found to be efficient in terms of power consumption and delay.
\end{abstract}

Keywords: Healthcare, body area networks (BAN), MAC, wakeup radio, power efficiency, lifetime

\section{Introduction}

Technological advancements have reduced cost, size, and affordability of sensor devices. This has helped expand the application area for sensor networks [1]. Healthcare is one of the major fields employing sensor devices and networks. The applications $[2,3]$ include both medical and non-medical. The medical applications are either wearable or implanted. Wearable devices can be used on the body surface, or at a very close proximity to the user. The implantable medical devices are inserted inside human body. Non-medical applications include file transfer and real-time audio/video streaming. A brief application summary is presented in Table 1.

Applications of sensor networks in healthcare nowadays are collectively called as body area networks (BAN). A typical BAN structure is shown in Figure 1. A BAN is heterogeneous in nature and needs to support diverse functionalities. It may include many devices and applications and has the characteristics of general wireless sensor networks. The devices are usually equipped with limited power, memory, and processing capabilities.

\footnotetext{
* Correspondence: kskwak@inha.ac.kr

UWB Wireless Communications Research Center, 6-142, Inha University, Yunghyeon-dong, Nam-gu, Incheon, Korea
}

A BAN can use the traditional approaches available for general sensor networks and must also have support to handle life-threatening emergency situations. A typical BAN may consist of few devices (called BAN nodes or $\mathrm{BNs}$ ) with option to add more devices as required. A BAN is managed and coordinated by a central device called the BAN network controller (BNC).

The major requirements for BAN [3] are low power consumption (power efficiency), low latency, scalability, quality of service (QoS), reliability, efficient bandwidth utilization, throughput, co-existence with other BANs, and high security. A BAN has different types of traffic. It needs to handle emergency and life critical situations. The devices in a BAN can be heterogeneous, and the data rate may vary from few $\mathrm{kb} / \mathrm{s}$ to few $\mathrm{Mb} / \mathrm{s}$. A BN can be used as wearable, implant, or at close proximity to the body. Similarly, BNs may operate at multiple frequency bands and hence support for multiple physical layers (PHYs) is important. The general topology in a BAN is star but may also have support for point to point communication. A BAN usually has small network size and is scalable to support new devices. Since not all the BNs need to send data all the time, they can be in the idle state and hence can be put into the sleep state to conserve power. Communications are bidirectional, from 
Table 1 Some BAN device and applications

\begin{tabular}{llll}
\hline $\begin{array}{l}\text { Application } \\
\text { type }\end{array}$ & Device & $\begin{array}{l}\text { Power } \\
\text { consumption }\end{array}$ & $\begin{array}{l}\text { Data } \\
\text { rate }\end{array}$ \\
\hline Implanted & Endoscope capsule & Low very & Medium \\
& $\begin{array}{l}\text { Deep brain stimulator } \\
\text { Drug delivery capsule }\end{array}$ & Low & Low \\
& $\begin{array}{l}\text { Brain liquid pressure } \\
\text { sensor }\end{array}$ & Very low & Low \\
& Glucose sensor & Vow low & Low \\
Wearable & ECG & Low & Low \\
& EMG & Low & Low \\
& EEG & Low & Low \\
& Blood pressure monitor & Low & Low \\
& Hear rate monitor & Low & Low \\
& pH monitor & Low & Low \\
& SpO2 & Low & Low \\
& Mp3 player & High & Medium \\
& MP4, video player & High & High \\
& Games & Hgih & High \\
\hline
\end{tabular}

$\mathrm{BNC}$ to $\mathrm{BN}$ and vice versa. A BAN may also have support to connect to other public networks via the Internet. This can occur when a person in a remote area needs to send data to some other place, e.g., a patient at home may need to send data to his or her doctor, who is away or in a different location. The BNC in this case can act as a gateway between BAN and external network.

The IEEE WPAN committee formed IEEE 802.15.6 Task Group 6 (TG6) to address the issues and standardize BAN. It intends to address both medical/healthcare and non-medical applications with diverse requirements. The media access control (MAC) layer in the standard intends to define a short range, wireless communication

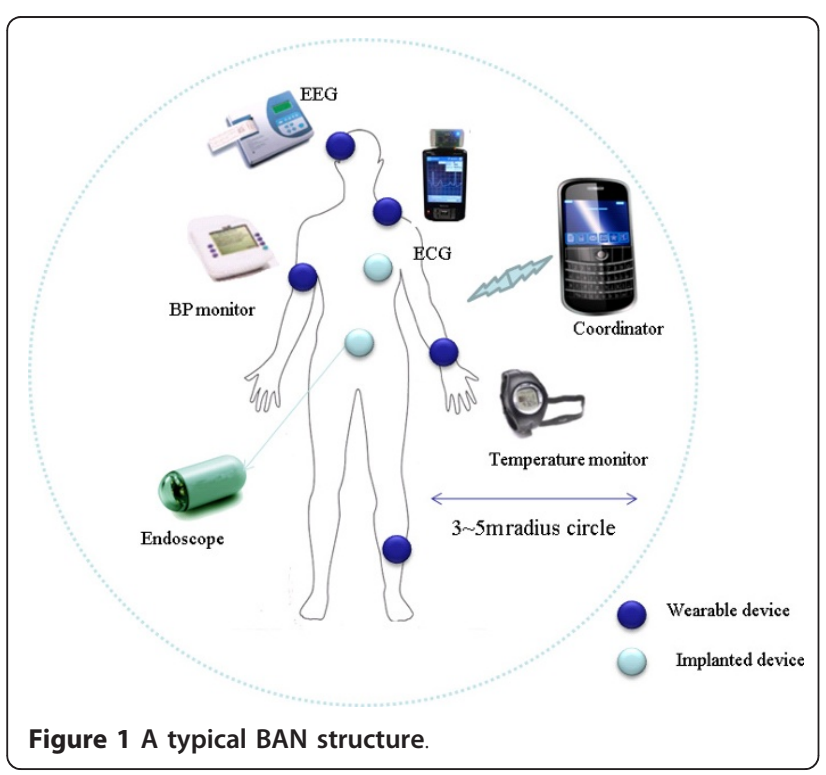

in and around the body area. The standard aims to support a low complexity, low cost, ultra-low power, and highly reliable and secure wireless communication for use in close proximity to, or inside, a human body (but not limited to humans) to satisfy an evolutionary set of entertainment and healthcare products and services. In this article, we propose a new MAC protocol for BAN using an out-of-band wakeup radio. A special wakeup radio circuit attached with each node is used to trigger a node to wakeup from sleep state.

The rest of the article is organized as follows. In Section 2 , we discuss the wakeup radio concept and the motivation behind our works. In Section 3, we describe the techniques and methodology used in our proposal. In Section 4, we explain the wakeup and communication processes along with the scheduling mechanisms. In Section 5, we present a detail analysis and discuss the results. Finally, the conclusions are drawn in Section 6.

\section{Wakeup radio concept and motivation}

\subsection{Wakeup radio}

A wakeup radio is a special circuit that is connected to the sensor device. The basic purpose of this circuit is to allow the main radio of the node to be in off state when there is no data communication takes place. The wakeup radio receiver can detect signals and generate an interrupt to wakeup the main radio. A wakeup radio allows a device to sleep and be woken up by suitable transmissions from another device.

The wakeup radio concept is explored and used in sensor networks. Authors of [4] have shown that radio-triggered hardware component can be used in sensor devices. The implementation is possible with very low cost. It is observed that the wakeup radio signal contains enough power to trigger a wakeup process. The wakeup radio can use its own separate antenna or share it with the main radio of the device. Figure 2 shows the schematic of a simple wakeup radio circuit and sensor device $[4,5]$.

The power consumption of some of the wakeup radio transceiver is presented in Table 2. The cost of adding extra wakeup radio circuit is very low. The circuit also does not use power from the main battery source of the sensor device. Hence, it does not incur an extra cost on power consumption.

There are many benefits of using a wakeup radio. The major advantages are

- Wakeup radio hardware is simple to design and implement

- Extracts energy from the radio signals

- Provides wakeup signals to the network node without using internal power supply

- Does not respond to normal data communication, and does not prematurely wakeup the sleeping node 
Table 2 Power consumption of various wakeup radio

\begin{tabular}{ll}
\hline Wakeup radio model & Power required $(\boldsymbol{\mu W})$ \\
\hline Basic [4] & 84 \\
Van doorn [24] & 171 \\
Ansari [23] & 12.53 \\
On CMOS [6] & 75 \\
\hline
\end{tabular}

A simple wakeup radio has limited range, typically 10 $15 \mathrm{ft}$. However, this makes itself suitable for short-ranged applications such as BAN. Another argument in favor of using out-of-band wakeup radio is that a wakeup radio-based scheme in BAN can save a significant amount of power.

\subsection{Motivation}

A BAN needs efficient handling of resources. To maintain a high performance and smooth flow in the network, it should be as hassle-free as possible in terms of operations. Power saving and low delay are the important factors. Hence, we evidently think that a MAC protocol for BAN should consider the following design issues:

- Minimize power consumption to increase the lifetime of the nodes

- Maximize sleep time for a node

- Minimize unnecessary wakeup periods to save power

- Minimize overheads (e.g. control packets overheads) in the network

- Minimize idle listening time

- Minimize collision and retransmission of a packet

- Minimize delay

- Efficient and quick response to emergency situations with minimum delay

Our aim is to develop a MAC protocol for BAN that can address these issues. A wakeup radio-based system can efficiently address the above issues. The wakeup radio can be used to wakeup a device only when necessary through a centrally coordinated system. In this system, the unnecessary wakeup periods for a device can be avoided thereby minimize the power consumption and increase the lifetime of the devices. A wakeup radio-based system through the on-demand request can significantly reduce the idle state power consumption. A device can remain in the sleep state until it is required to transmit data. This can be pre-programmed in the controller device. Whenever required, a wakeup radio signal can be sent to switch on the device. In our study, we propose to use a very low-powered wakeup signal to trigger the wakeup circuit integrated with the BNs.

Collision is one of the major problems in any sensor network. It should be avoided, as retransmission leads to extra power consumption and delay. The on-demand wakeup mechanism proposed in this study can help avoid collisions, and delay. It also reduces the extra overheads that are employed to avoid collisions in normal MAC protocols for WSN. For example, in a CSMA/CA base protocol, a lot of control packets (request to send packet-RTS-by a sender, clear to send packet-CTS-by a receiver) are generated to complete a successful data communication. A wakeup radio can help to reduce these packet overheads. An emergency situation in medical applications should be promptly handled. A wakeup radio can be used to send emergency command to the controller efficiently.

Power efficiency is the dominant factor in designing and implanting a wireless sensor network. MAC protocols in WSN aim to reduce power consumption and delay. Due to vast nature of mechanisms and techniques used in these MACs, a smooth classification of MAC protocols is not an easy task. The major design factors are application specific. A wireless sensor node requires an ultra-low power RF transceiver in order to meet the stringent power requirements of the system. Since the transceiver consumes power whenever it is active, it would be advantageous to leave it off and wake it up only when a packet is being transmitted or received [6]. It is an established fact that power consumption in the sleep state is very less compared to the idle state. Hence, the majority of MACs try to take advantage of sleep (radio off) state. A node can undergo on/off cycle. This technique, known as the duty cycle, is very popular
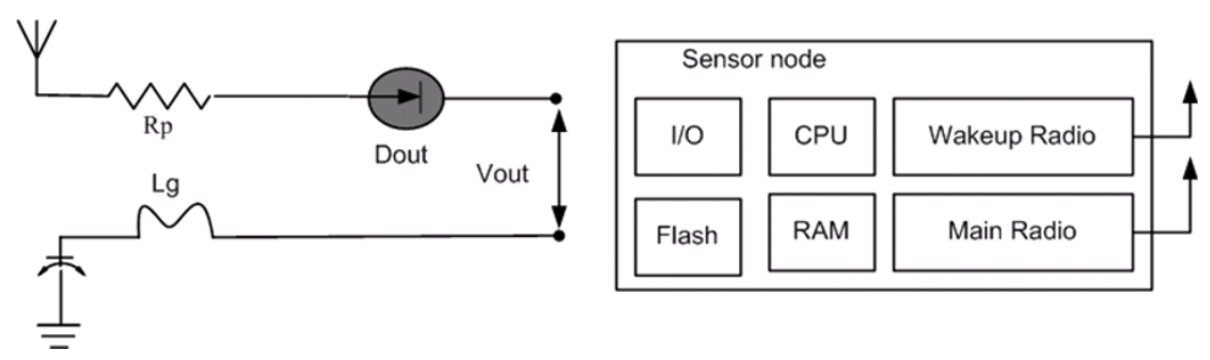

Figure 2 Wakeup radio: (a) A basic wakeup radio circuit; (b) Schematic of a sensor node using wakeup radio module. 
in WSN. A node can also remain in off state until it is woken up by an out-of-band radio mechanism. It is commonly known as the on-demand mechanism.

Duty cycle is one of the most widely used techniques in MAC protocols. Duty cycle MACs use radio ON/OFF technique to reduce idle listening. Duty cycle MACs can be broadly classified into two major categories:

- Fixed duty cycle MACs

- Adaptive duty cycle MACs

A fixed duty cycle MAC uses fixed length period. Some of the earliest MAC protocols (e.g., S-MAC [7]) are based on the fixed duty cycle concept. In adaptive duty cycling MACs such as T-MAC [8] and WiseMAC [9], the sleep/wakeup time of sensor nodes is adaptively determined. They are more effective in saving power than the fixed non-adaptive MAC protocols. Duty cycle MACs can be further classified as follows:

- Synchronous duty cycle MACs

- Asynchronous duty cycle MACs

Synchronous MACs need time synchronization before data communication while asynchronous MACs such as B-MAC [10], X-MAC [11] are independent of such a requirement. Although these protocols are effective, they have their own disadvantages. It remains to be seen if they can satisfy the application requirement of BAN. The duty cycle-based MACs have to deal with issues such as idle state power consumption, collision, overhearing of packets that are not intended to it and packet overheads.

Another more effective way is to use an on-demand mechanism employing the wakeup radio. An additional ultra-low power receiver is attached to the sensor nodes that can help save a significant amount of power by minimizing the idle listen period for the main radio. Out-of-band radio mechanism has been proposed for sensor networks to minimize power consumption [4,5,12-15]. All of these works use an extra radio channel for waking up the sensor devices.

The wakeup radio-based MAC proposed in this study is an on-demand MAC. To evaluate and compare our work, we have chosen some of the well known and popular MAC protocols such as B-MAC, X-MAC, WiseMAC, and ZigBee. Berkeley MAC (B-MAC) is an asynchronous MAC protocol with an adaptive duty cycling mechanism. It duty cycles through a periodic channel sampling called low power listening (LPL). It uses the LPL or channel sampling scheme to link to a receiver. It employs an improved filtering mechanism to increase the reliability of channel assessment. Nodes periodically wakeup for a short time interval in each duty cycle to check for preamble. The preamble is long enough for a receiver to detect it. When a node wakes up, it turns on the radio and checks for activity in the channel. It uses the clear channel assessment (CCA) technique to detect activity. If any activity is detected, the node stays awake for the time period required to receive the packet or else a timeout puts it back to sleep state. Once the reception is completed, the node goes back to sleep mode. X-MAC is a low power, asynchronous MAC protocol. The primary goal of X-MAC is to overcome the extra latency and extra power consumption at non-target neighboring nodes unlike MACs that uses long preambles (e.g., BMAC). It uses a preamble to inform the neighbor node about imminent data communication but avoids the long preamble through a concept of short strobe preambles. The strobe preambles contain the destination node ID. In between two strobe preambles, it awaits for an early acknowledgement from the receiver. WiseMAC is based on the preamble sampling to minimize idle listening and save power. It uses a CSMA base technique for multiple access. Nodes sample the medium with a constant period at a regular interval. They also listen to the channel for a short duration of time. The IEEE 802.15.4 ZigBee network includes a central coordinator which acts as an access point. It is also considered as a candidate MAC for BAN. Authors of [16-18] have proposed and evaluated ZigBee for BAN.

CSMA and TDMA are the most common techniques used in a sensor network MAC. CSMA/CA is used for contention-based protocols. Majority of the CSMA/CA base MAC uses RTS and CTS packets before data communication. This causes lots of packet overheads. Use of wakeup radio can minimize the extra power consumption by the RTS-CTS packet exchange which is done by the main radio. TDMA is popular in synchronous MACs. Authors of [19-21] have proposed TDMA base MAC protocols for BAN. A TDMA-based scheme combined with wakeup radio can be used to design a power efficient MAC. A TDMA-based approach has many advantages over other similar techniques such as CSMA/CA and FDMA. Authors of [12] have provided a comparison between TDMA and CSMA/CA as shown in Table 3. In our study, we propose to use TDMA for the multiple access mechanism.

\section{Table 3 Comparison between TDMA and CSMA/CA}

\begin{tabular}{lll}
\hline Parameters & TDMA & CSMA/CA \\
\hline Power consumption & Low & High \\
Bandwidth utilization & Maximum & Low \\
Preferred traffic level & High & Low \\
Dynamic (network change) & Poor & Good \\
Effect of packet failure & Latency & Low \\
Synchronization & Crucial & Not crucial \\
\hline
\end{tabular}




\section{Proposed model and methodology}

In this section, we discuss our proposed work in detail. First, we discuss the traffic and device classification to be used in our model followed by device states.

\subsection{Traffic assumption}

A typical BAN consists of heterogeneous traffic. Traffic is generated by both BNs and BNC. In our model, we have used two basic priorities: periodic or normal and random traffic or emergency. Periodic traffic happens when communication is initiated by $\mathrm{BNC}$ based on predefined schedule. Emergency traffic is generated when a node needs to send urgent data. Sudden heart rate or blood pressure increase or decrease can be considered as emergency traffic. This kind of traffic is totally random and unpredictable.

\subsection{BAN devices and states}

There are different types of devices in a BAN. These devices can be classified into two major categories: full function device (FFD) and reduced function device (RFD). Hence, a typical BN can either be FFD or RFD. A $\mathrm{BN}$ can act and respond according to instructions from BNC. BAN device and traffics are shown in Table 4.

A FFD device can perform a complex task and is equipped with support for management, control and data transfer functions. They have support for multiple PHYs. They have wakeup radio transceivers and have support for point to point communication and able to communicate with other BNs in a different BAN. A FFD device can act as BNC. AN RFD device is much simpler and has fewer functionalities than an FFD device. They are application specific and mainly focus on data communication. They can receive wakeup radio signals from the BNC. Device classification is shown in Figure 3.

In our model, a $\mathrm{BN}$ can be in any of the two major states: sleep state and wakeup state. Sleep state is the default state. A BN goes to wakeup state to communicate. A wakeup state is transitioned into the idle state (ready state). From idle state, a BN goes to either transmit $(\mathrm{Tx})$ state or receive $(\mathrm{Rx})$ state as per the need. When communication is not required, a BN can directly go to sleep state from idle state. The state transition diagram of BAN device is shown in Figure 4.

Table 4 BAN traffics and wakeup radio

\begin{tabular}{lll}
\hline Device & Periodic traffic & Random traffic \\
\hline BN & $\begin{array}{l}\text { Receives wakeup radio signal } \\
\text { from BNC }\end{array}$ & $\begin{array}{l}\text { Sends a wakeup radio signal } \\
\text { to BNC }\end{array}$ \\
BNC & Sends wakeup radio signal to & $\begin{array}{l}\text { Receives wakeup radio signal } \\
\text { from BN }\end{array}$ \\
\hline
\end{tabular}

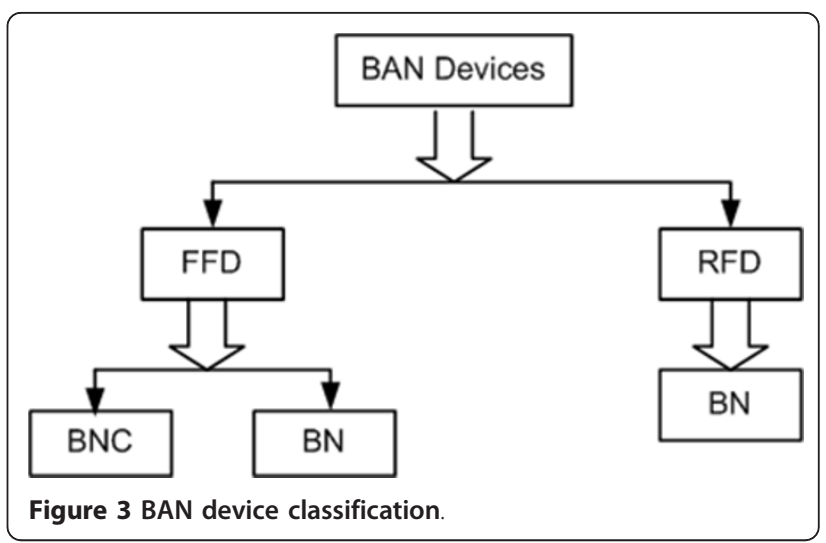

\section{Wakeup process and scheduling mechanisms}

A wakeup process is handled using wakeup radio and is coordinated and managed by the BNC. A two-stage communication process is proposed. The generalized process can be summarized as shown in Figure 5 .

In stage 1 , the wakeup radio part of the $\mathrm{BN}$ is switched $\mathrm{ON}$. This can happen when a $\mathrm{BN}$ receives the wakeup radio signal from the $\mathrm{BNC}$. If the $\mathrm{BN}$ verifies itself as the intended receiver, it sends an acknowledgement to the BNC as a reply in the same channel and proceeds to stage 2 . In stage 2 , the main radio transceivers are triggered $\mathrm{ON}$ for data communication. The flow chart is shown in Figure 6. Wakeup radio is used to trigger a wakeup in the BN. After a successful wakeup, a beacon is sent by $\mathrm{BNC}$ with relevant information. Communication takes place in guaranteed time slots (GTS) as discussed in later section. If the BN

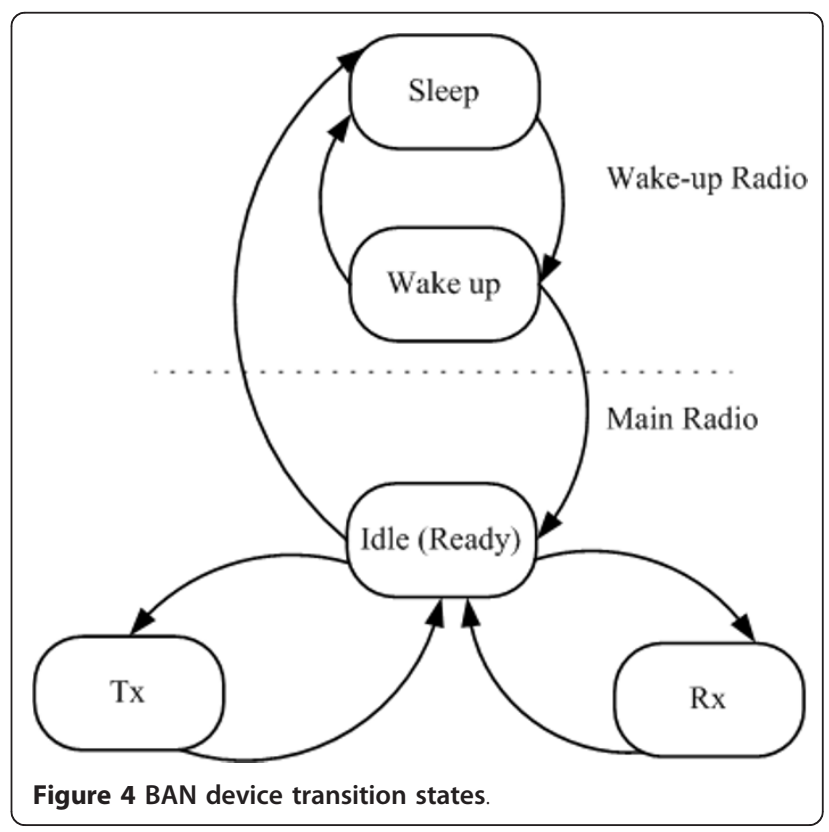




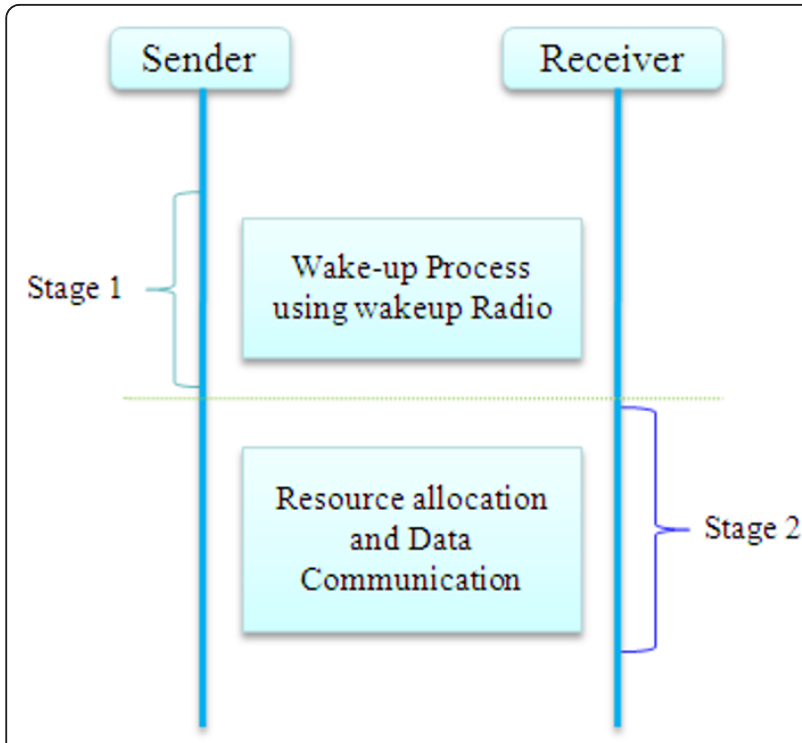

Figure 5 Communication process using wakeup radio

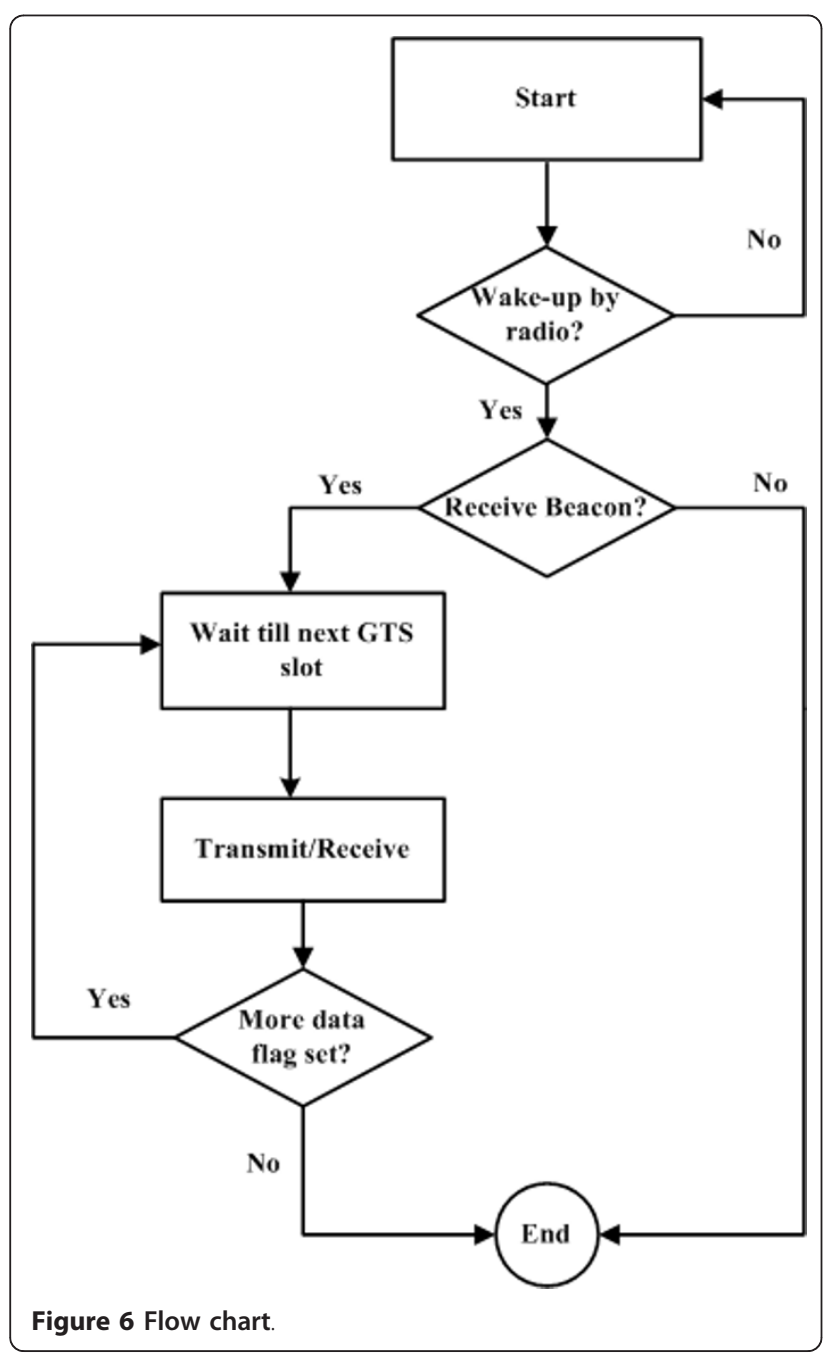

needs to send more data, it sets the flag in the MAC data frame.

\subsection{Wakeup scheduling for periodic traffic}

A wakeup mechanism based on traffic intensity at each $\mathrm{BN}$ is used for communication in case of normal traffic. $\mathrm{BNC}$ maintains the wakeup schedule in a table for every node in the network which is constructed based on traffics at the particular BN. Wakeup interval is calculated from inter-arrival of packets for a BN. Use of wakeup table by BNC saves a significant amount of power as all $\mathrm{BNs}$ in the network remain in the sleep state (i.e., switch off the main transceiver) until it is woken up by the BNC.

The inter-arrival parameters $i, j, \ldots, q$ listed in Table 5 are reconfigurable values for each $\mathrm{BN}$. For example, in case of a patient, a doctor/nurse or in-charge person can set the packet inter-arrival time for temperature monitor (node BN-008) to be $6 \mathrm{~h}$ or 21,600 s. This will cause the BNC to send a wakeup radio signal to the particular BN after the specified intervals and complete the data communication. The $\mathrm{BN}$, between two consecutive wakeup periods can switch off its main radio and go to sleep state to save power. It does not have to contend for data communication. The inter-arrival time can be reset and reconfigured as the need arises. A node like heart rate monitor can have very low inter-arrival time (e.g., $100 \mathrm{~ms}$ ) for a particular amount of time such as exercise or operations. It can be reset again to match other desired level of data communication. We have assumed that every node in the network uses wakeup radio with $\mathrm{BNC}$ managing a wakeup scheduling table.

The data transmission starts in a specific time slot and ends with an acknowledgement (Ack) from the BNC as shown in Figure 7. If a $\mathrm{BN}$ is woken up by $\mathrm{BNC}$ but has no data at the current moment, it sends a negative acknowledgement (NAck) to BNC, and further communication does not take place.

\subsection{Wakeup scheduling for random (emergency) traffic}

Wakeup radio is also used to handle an emergency situation. Emergency events are random and totally

Table 5 Wakeup schedule table

\begin{tabular}{lll}
\hline BAN nodes & Node Id & Inter-arrival time (s) \\
\hline Heart rate monitor & $\mathrm{BN}-001$ & $i$ \\
BP monitor & $\mathrm{BN}-002$ & $j$ \\
Glucose monitor & $\mathrm{BN}-003$ & $k$ \\
SpO2 & $\mathrm{BN}-004$ & $l$ \\
$\mathrm{EEG}$ & $\mathrm{BN}-005$ & $m$ \\
$\mathrm{ECG}$ & $\mathrm{BN}-006$ & $n$ \\
pH monitor & $\mathrm{BN}-007$ & 0 \\
Temperature monitor & $\mathrm{BN}-008$ & $p$ \\
Insulin pump & $\mathrm{BN}-009$ & $q$ \\
\hline
\end{tabular}




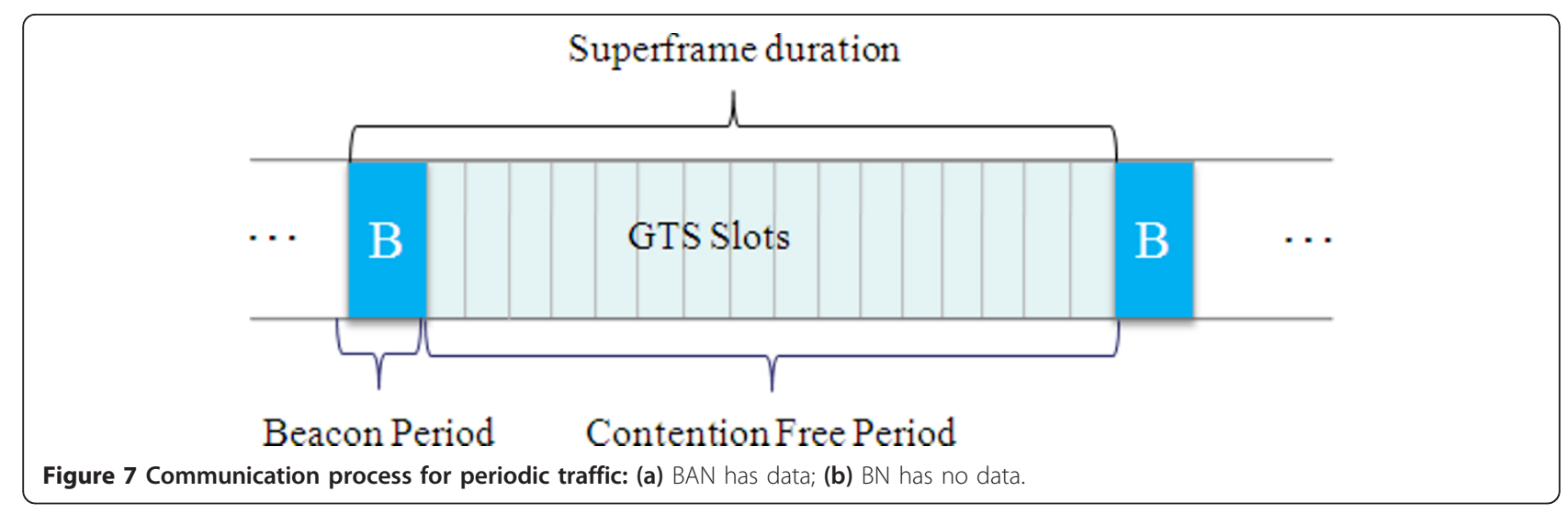

unpredictable. Emergency data communication happens when some emergency event occurs and a BN suddenly wakes up to send data to BNC. The schematic is shown in Figure 8. Let us assume a scenario when a $\mathrm{BN}$ has some emergency data to be sent to the BNC. In this case, a BN wakes up and sends a wakeup radio to the BNC. BNC acknowledges it by sending an Ack packet.
It sends the beacon to the $\mathrm{BN}$ for resource allocation. The $\mathrm{BN}$ sends the data to BNC. Communication ends when $\mathrm{BNC}$ sends Ack to the $\mathrm{BN}$.

\subsection{Resource allocation}

We have used the TDMA scheme for multiple access and resource allocation. The BNC is responsible for the

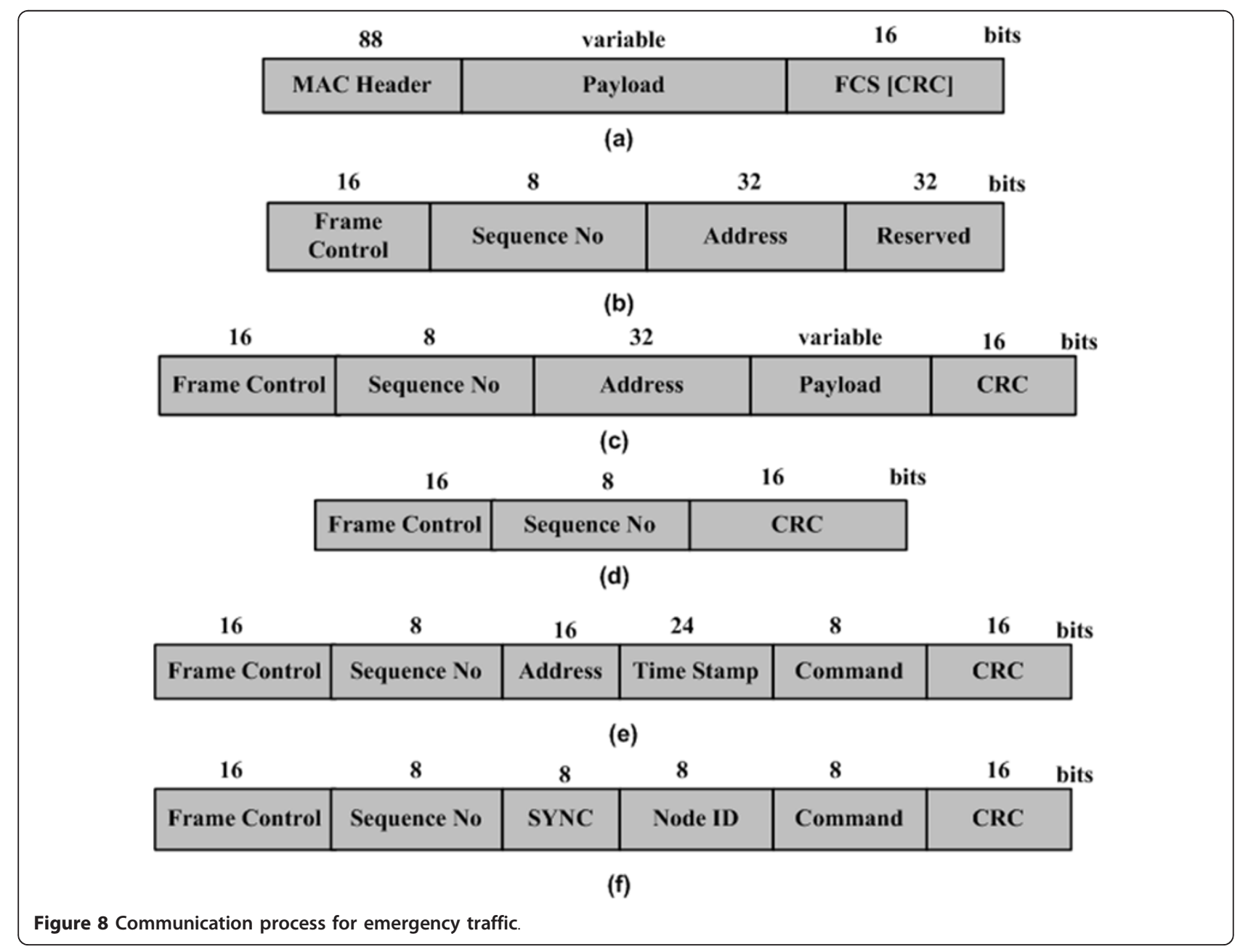




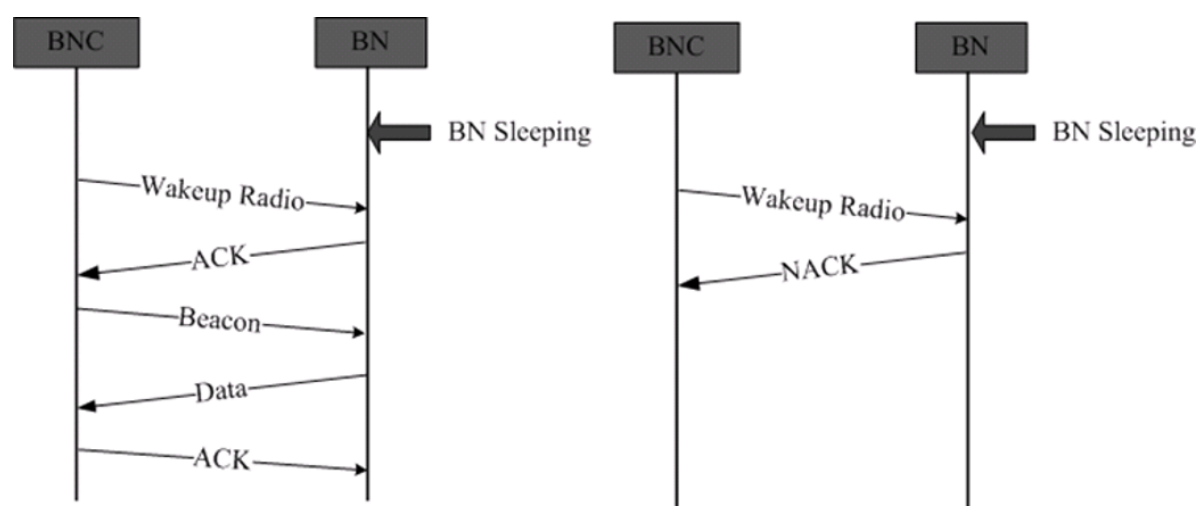

Figure 9 Superframe structure.

channel and slots allocation. For a successful communication, BNC allocates an available channel and then the time slot to a BN. Channel allocation is done through the use of a special field in the beacon frame. 'Channel and GTS slots Allocation Table' is maintained by the BNC. A superframe structure as shown in Figure 9 is used. The superframe contains a Beacon period and contention-free period (CFP). The CFP contains 15 GTS slots. Since the $\mathrm{BNC}$ knows the wakeup patterns of each $\mathrm{BN}$, it sends the wakeup radio signal to wakeup a $\mathrm{BN}$ from sleep state to wakeup state. The BN sends an acknowledgement (Ack packet) to the BNC indicating it is now awake. The BNC then sends the beacon to the node. The BN grabs the beacon that contains synchronization, priority, channel, and slot information.

The MAC frames are shown in Figure 10. The MAC frame, MAC header, data, Ack, beacon, and wakeup frames are shown.

\section{Analysis and simulation}

Periodic/normal traffic is generated using wakeup table. We have used the Poisson model for emergency events. Since we only need to wakeup a BN randomly for emergency data communication, Poisson model sufficiently serves the purpose.

\subsection{Analysis}

The variables used in this study are listed in Table 6.

We have used the following general expression (1) to calculate average power consumption.

$$
P_{\text {avg }}=P_{\text {avg-wk }}+P_{\text {avg-tx }}+P_{\text {avg- } r x}+P_{\text {avg-ov }}
$$

where $P_{\text {avg }}$ is the average power consumed. $P_{\text {avg-wk }}$ $P_{\text {avg-tx }}$, and $P_{a v g-r x}$ are the average power consumed in wakeup, transmit, and receive states, respectively. $P_{\text {avg-ov }}$ is the average overhead power consumption.

\subsection{1. $B-M A C$}

It uses the asynchronous duty cycle mechanism and sends a long continuous preamble for communication as shown in Figure 11. It performs CCA before communication.

The average power consumption for preamble for BMAC is calculated using (2).

$$
P_{\text {avg-wk }}=P_{t x} \times \frac{T_{p}}{T}
$$

The BN transmits the data packet along with the long preamble and waits for the Ack from the receiver node. The average power consumption for transmitting the

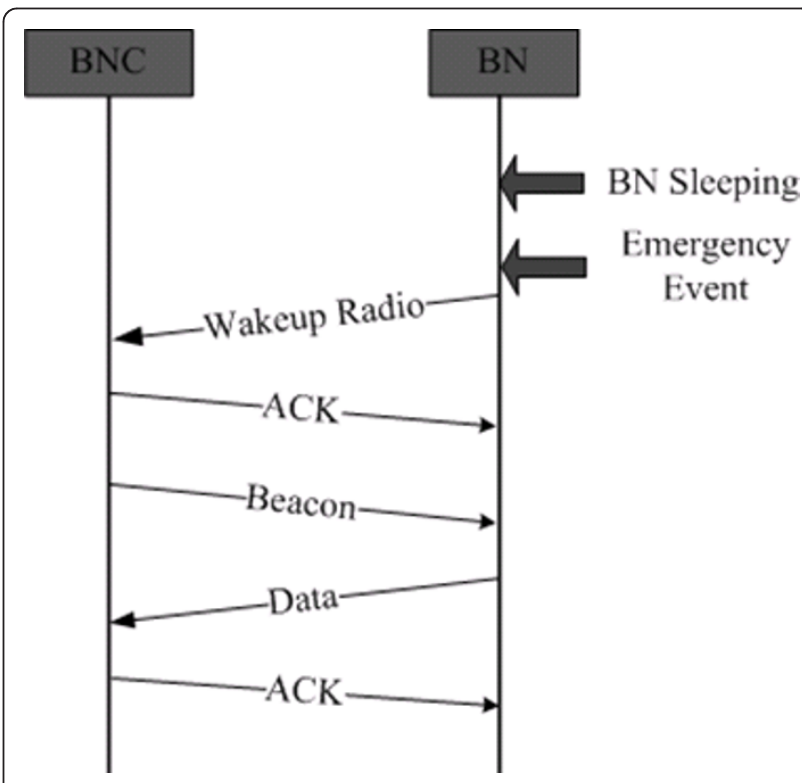

Figure 10 MAC frames: (a) MAC frame; (b) MAC header; (c) data; (d) Ack; (e) beacon; (f) wakeup frame. 


\section{Table 6 List of variables}

\begin{tabular}{|c|c|}
\hline Variable & Explanation \\
\hline$P_{t x}$ & Power required for TX (transmission) \\
\hline$P_{r x}$ & Power required for RX (reception) \\
\hline$T_{\text {data }}$ & Time required for data packet transmission \\
\hline$T_{\text {ack }}$ & Time required for Ack packet transmission \\
\hline$T_{b}$ & Time required for Beacon packet transmission \\
\hline$P_{t}$ & Power required to switch the transceiver between $\mathrm{RX}$ and $\mathrm{TX}$ \\
\hline$T_{t}$ & Time required to switch the transceiver between $\mathrm{RX}$ and $\mathrm{TX}$ \\
\hline N & Number of one hop neighboring nodes \\
\hline$P_{\text {active }}$ & Power consumption in active state \\
\hline$R$ & Data rate of the radio \\
\hline$L_{x}$ & Length of a frame $x$, where $x \in\{$ data, ack, beacon, wakeup $\}$ \\
\hline$P_{t r}$ & $\begin{array}{l}\text { Power required to turn on the transceiver from sleep state } \\
\text { into the RX or TX state }\end{array}$ \\
\hline$T_{t r}$ & $\begin{array}{l}\text { Time required to turn on the transceiver from sleep state into } \\
\text { the } \mathrm{RX} \text { or TX state }\end{array}$ \\
\hline$\theta$ & Crystal tolerance of a wakeup timer \\
\hline$T_{\text {cсa }}$ & Time required for CCA or carrier sensing \\
\hline$P_{s l}$ & Power consumed in sleep state \\
\hline$P_{t w b n}$ & Power required to transmit low power wakeup packet \\
\hline$P_{\text {rwon }}$ & Power required to receive low power wakeup packet \\
\hline$T_{\text {won }}$ & Time required to send low power wakeup packet \\
\hline$P_{\text {twack }}$ & Power required to transmit low power Ack packet \\
\hline$T_{w k}$ & Interval between preamble/beacon samplings \\
\hline$T_{s l}$ & Time spend in sleep state \\
\hline$T_{p}$ & Preamble duration \\
\hline$T_{\text {wrrx }}$ & Interval between preamble samplings in X-MAC \\
\hline Nprobe & Expected preamble-listen iterations required \\
\hline$T$ & Total time duration \\
\hline$T_{p x}$ & Average number of preambles during time $T$ \\
\hline
\end{tabular}

data packet and receiving the Ack packet can be calculated using (3) and (4).

$$
\begin{aligned}
& P_{\text {avg-tx }}=P_{t x} \times \frac{T_{d a t a}}{T} \\
& P_{a v g-r x}=P_{r x} \times \frac{T_{a c k}}{T}
\end{aligned}
$$

Power consumed due to other overheads includes sleep power, transition power, turnaround power, CCA and overhearing power by other nodes. The overhearing power $\left(P_{o h}\right)$ can be calculated using $(5)$.

$$
P_{o h}=P_{r x} \times \frac{\left(\frac{T_{w k}}{2}-T_{c c a}\right)+T_{d a t a}}{T} \times(N-1)
$$

The average overhead power consumption can be calculated using (6).

$$
\begin{aligned}
P_{\text {avg-ov }} & =P_{s l} \times \frac{T_{s l}}{T}+P_{r x} \times \frac{\left(T_{t r}+T_{c c a}\right)}{T}+P_{t} \times \frac{T_{t}}{T}+P_{t r} \times \frac{T_{t r}}{T} \\
+P_{r x} & \times \frac{\left(\frac{T_{w k}}{2}-T_{c c a}\right)+T_{d a t a}}{T} \times(N-1)+P_{r x} \times \frac{T_{p x}}{T}
\end{aligned}
$$

Equation (7) shows the total sleep time $\left(T_{s l}\right)$.

$$
T_{s l}=T-\left(T_{t r}+T_{p}+T_{t}+T_{d a t a}+T_{a c k}+T_{p x}\right)
$$

where

$$
T_{p x}=T_{c c a} \times \frac{T}{T_{w k}}
$$

\subsection{2. $X-M A C$}

It uses short strobe preamble. On an average, it spends two preambles and one Ack packet, $\left(2 P_{p}+T_{a c k}\right)$ time on channel polling, where $\left(T_{p}\right)$ is the preamble time and $\left(T_{a c k}\right)$ is the Ack packet time. Mechanisms of short preamble based X-MAC is shown in Figure 12.

The average power consumption for preamble for $\mathrm{X}$ MAC is calculated using (8).

$$
P_{a v g-w k}=N_{\text {probe }} \times \frac{\left(P_{t x} \times T_{p}\right)+\left(P_{r x} \times T_{a c k}\right)}{T}
$$

The BN transmits the data packet along with the long preamble and waits for the Ack from the receiver node. The average power consumption for transmitting the data packet and receiving the Ack packet can be calculated using (9) and (10).

$$
P_{\text {avg-tx }}=P_{t x} \times \frac{T_{\text {data }}}{T}
$$

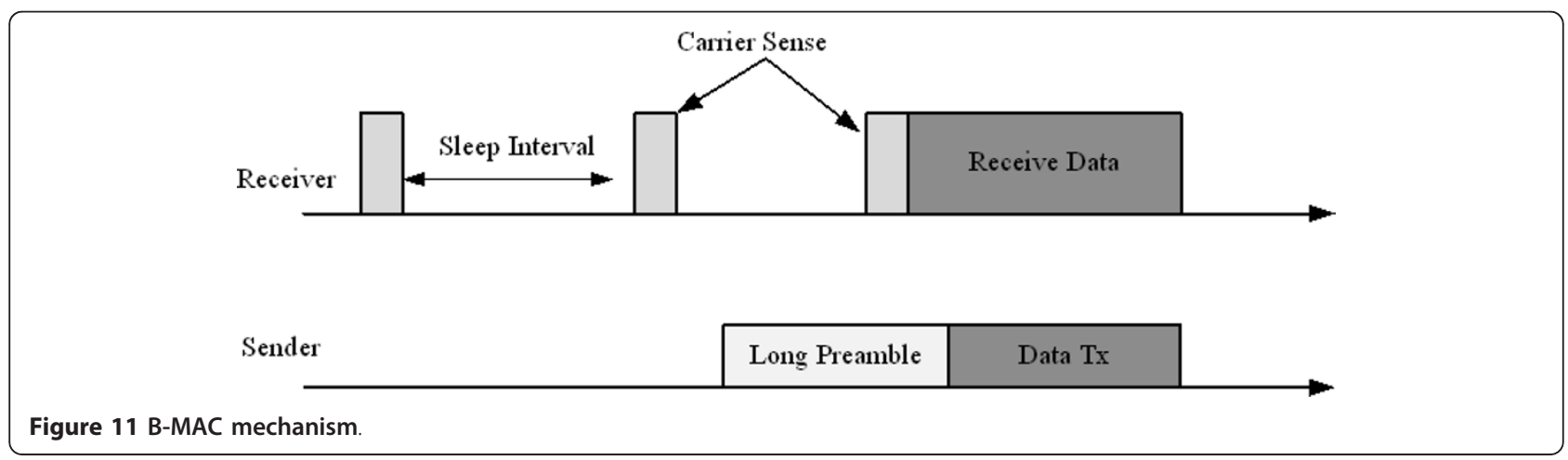




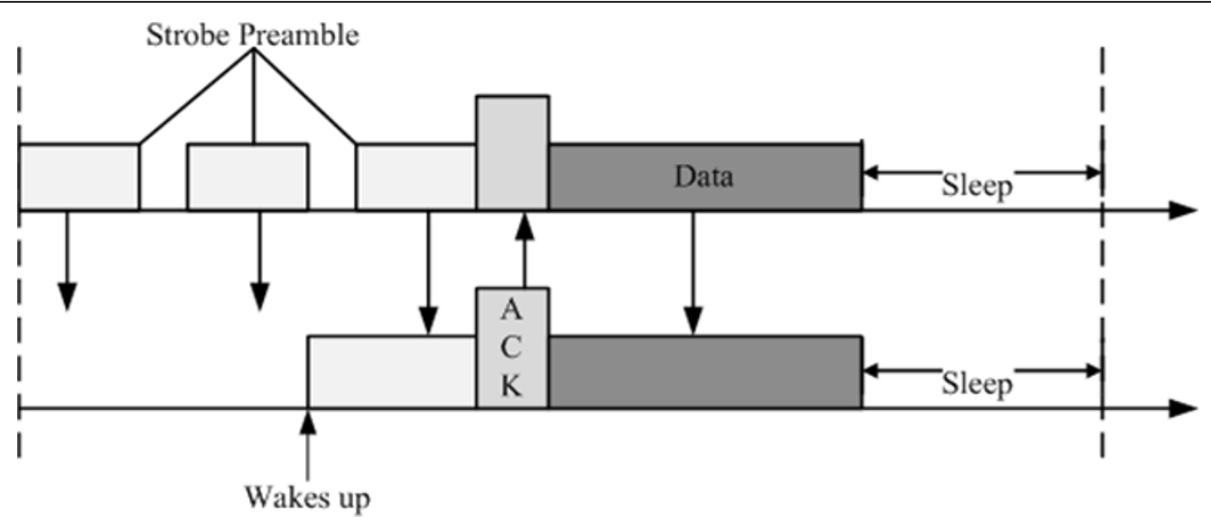

Figure 12 X-MAC mechanism

$$
P_{a v g-r x}=P_{r x} \times \frac{T_{a c k}}{T}
$$

Power consumed due to other overheads includes sleep power, transition power, turnaround power and overhearing power by other nodes. The overhearing power $\left(P_{o h}\right)$ can be calculated using $(11)$.

$$
P_{o h}=P_{r x} \times \frac{\left(2 T_{p}+T_{a c k}\right)}{T} \times(N-1)
$$

The average overhead power consumption can be calculated using (12).

$$
\begin{aligned}
& P_{a v g-o v}=P_{s l} \times \frac{T_{s l}}{T}+P_{t r} \times \frac{T_{t r}}{T}+P_{t} \times \frac{3 T_{t}}{T}+P_{r x} \times \frac{\left(2 T_{p}+T_{a c k}\right)}{T} \times(N-1) \\
& +P_{r x} \times \frac{T_{p x}}{T}
\end{aligned}
$$

Equation (13) shows the total sleep time $\left(T_{s l}\right)$.

$$
T_{s l}=T-\left(T_{t r}+N_{\text {probe }}+T_{p}+3 T_{t}+T_{\text {data }}+2 T_{a c k}+T_{p a z}\right)
$$

where

$$
\begin{aligned}
& T_{p x}=\left(2 T_{p}+T_{a c k}\right) \times \frac{T}{T_{w k r x}} \\
& N_{\text {probe }}=\frac{T_{w k r x}}{2\left(T_{p}+T_{a c k}\right)}
\end{aligned}
$$

\subsubsection{WiseMAC}

In WiseMAC, the access point (AP) learns the sampling schedule of all the sensor nodes. This helps the AP to start the transmission at the right moment with a wakeup preamble of minimized duration $T_{p}$. The AP regularly updates the sampling schedule information of all the sensor nodes. Initially, the size of the preamble is set to be equal to the sampling period. In a later period, it dynamically determines the length of the preamble by using the knowledge of the sleep schedules of the neighboring nodes. Mechanism of WiseMAC is shown in Figure 13.

The average power consumption for WiseMAC can be calculated using (14) to (19). The average power consumption for transmitting the data packet and receiving the Ack packet can be calculated using (15) and (16).

$$
\begin{aligned}
& P_{\text {avg-wk }}=P_{t x} \times \frac{\frac{1}{R}}{T_{w k}} \\
& P_{\text {avg-tx }}=P_{t x} \times \frac{\left(\bar{X}+T_{\text {data }}\right)}{T} \\
& P_{\text {avg-rx }}=P_{r x} \times \frac{T_{a c k}}{T}
\end{aligned}
$$

Power consumed due to other overheads includes sleep power, transition power, turnaround power and overhearing power by other nodes. The overhearing power $\left(P_{o h}\right)$ can be calculated using (17).

$$
P_{o h}=P_{r x} \times \frac{\bar{Y}}{T} \times(N-1)
$$

The average overhead power consumption can be calculated using (18).

$$
\begin{aligned}
& P_{\text {avg-ov }}=P_{s l} \times \frac{T_{s l}}{T}+P_{t r} \times \frac{T_{t r}}{T}+P_{t} \times \frac{T_{t}}{T}+P_{r x} \times \frac{\bar{Y}}{T} \times(N-1) \\
& +P_{r x} \times \frac{T_{p x}}{T}
\end{aligned}
$$

Equation (19) shows the total sleep time $\left(T_{s l}\right)$.

$$
T_{s l}=T-\left(T_{t r}+T_{t}+T_{d a t a}+T_{a c k}+T_{p x}+\frac{T_{w k}}{2}\right)
$$




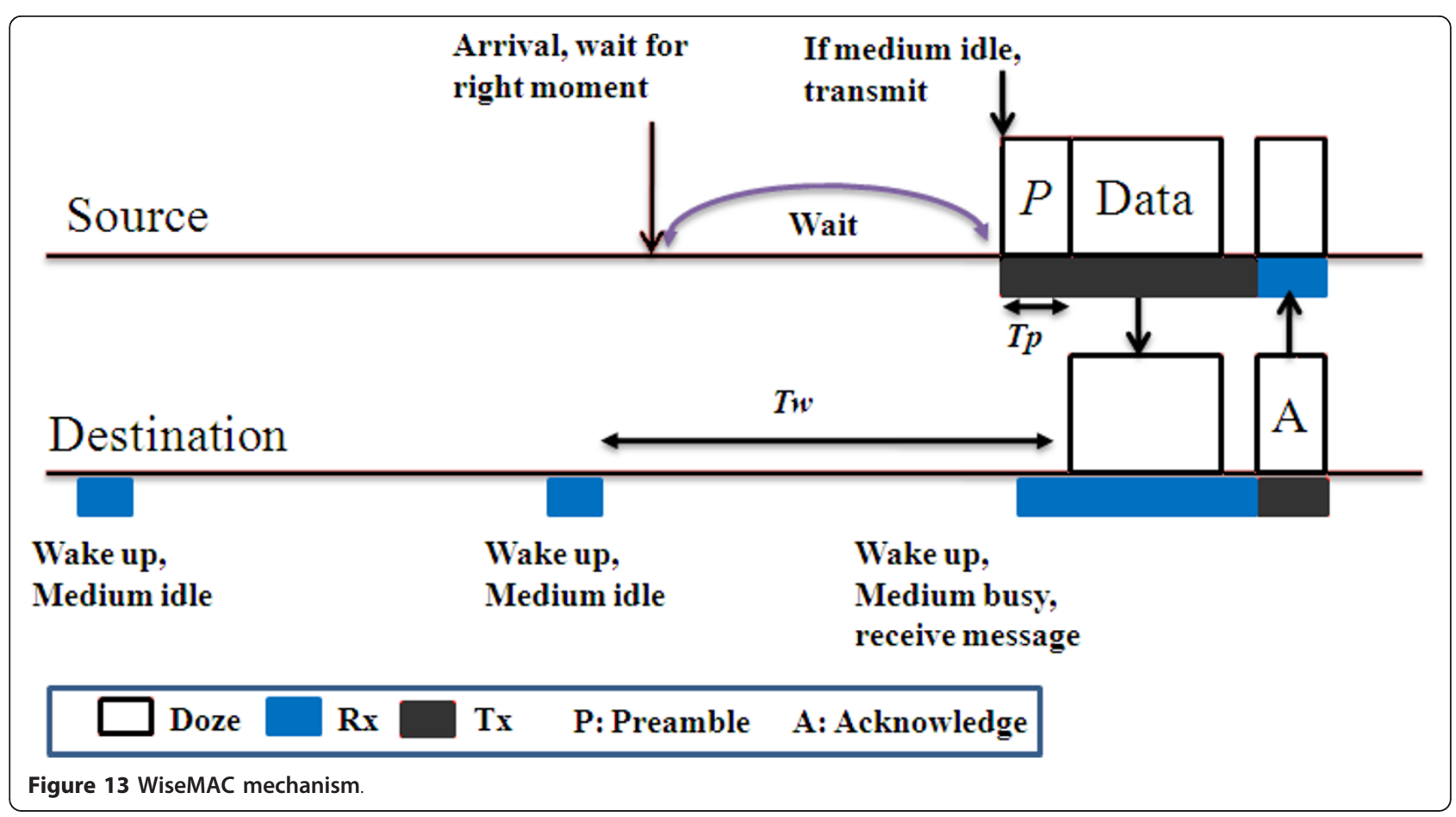

where

$$
\begin{aligned}
& T_{p x}=T_{p} \times \frac{T}{T_{w k}} \\
& \bar{X}=2 \theta L\left(1-e^{-\frac{T_{\text {data }}}{4 \theta L}}\right) \\
& \bar{Y}=\frac{T_{\text {data }}^{2}+12 \theta L T_{\text {data }}}{2 T_{w k}}\left(1-e^{-\frac{T_{w k}}{4 \theta L}}\right)
\end{aligned}
$$

$\bar{X}$ is the duration during which a destination sensor node listens to the wakeup preamble prior to detect the start of the first data packet. $\bar{Y}$ is the average duration during which a potential over-hearer listens to a transmission.

\subsubsection{ZigBee MAC}

The power saving mode of ZigBee as explained in [9] is considered for comparison with the proposed MAC protocol. It supports star topology as in the case of the proposed model too. To calculate the power consumption, we have taken the data communication scenario when a node uploads data to the coordinator. We have considered the beacon mode of ZigBee. A beacon is periodically transmitted with a period $T_{w k}$. A node listens for the beacon and then synchronization is done. The node transmits its data packet to the coordinator using
CSMA/CA scheme. The coordinator may acknowledge the successful reception of the data by transmitting an optional Ack packet as shown in Figure 14.

The average power consumption for ZigBee MAC can be calculated using (20) to (25). The average power consumption for transmitting the data packet and receiving the Ack packet can be calculated using (21) and (22).

$$
\begin{aligned}
& P_{a v g-w k}=P_{r x} \times \frac{\left(2 \theta T_{w k}+T_{b}\right)}{T} \\
& P_{a v g-t x}=P_{t x} \times \frac{T_{d a t a}}{T} \\
& P_{a v g-r x}=P_{r x} \times \frac{T_{a c k}}{T}
\end{aligned}
$$

Power consumed due to other overheads includes sleep power, transition power, turnaround power and overhearing power by other nodes. The overhearing power $\left(P_{o h}\right)$ can be calculated using $(23)$.

$$
P_{o h}=P_{r x} \times \frac{\left(2 \theta T_{w k}+T_{b}\right)}{T} \times(N-1)
$$

The average overhead power consumption can be calculated using (24).

$$
\begin{aligned}
& P_{a v y-o v}=P_{s l} \times \frac{T_{s l}}{T}+P_{t r} \times \frac{T_{t r}}{T}+P_{t} \times \frac{2 T_{t}}{T}+P_{r x} \times \frac{2 T_{c c a}}{T} \\
& +P_{r x} \times \frac{\left(2 \theta T_{w k}+T_{b}\right)}{T} \times(N-1)+P_{r x} \times \frac{T_{p x}}{T}
\end{aligned}
$$



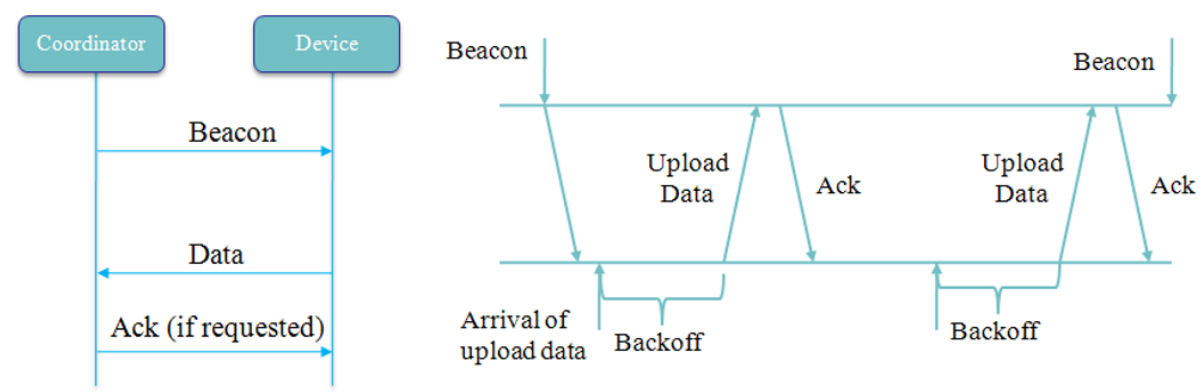

Figure 14 ZigBee MAC communication process

Equation (25) shows the total sleep time $\left(T_{s l}\right)$.

$$
T_{s l}=T-\left(T_{t r}+2 T_{t}+T_{d a t a}+T_{a c k}+T_{p x}+2 T_{c c a}\right)
$$

where

$$
T_{p x}=\left(2 \theta T_{w k}+T_{b}\right) \times \frac{T}{T_{w k}}
$$

\subsubsection{Proposed MAC}

In the proposed MAC, a BN sleeps until it is woken up by the BNC. The BNC sends a wakeup signal to wakeup the $\mathrm{BN}$. The intended receiver $\mathrm{BN}$ checks the address in the wakeup packet. If it matches, it triggers on the main radio for data communication. A beacon is received from BNC. The BN sends the data packet to the BNC and waits for the Ack.

Equation (26) is used to calculate the average wakeup power consumption for the proposed MAC. The receiver $\mathrm{BN}$ receives the wakeup radio packet and then transmits a wakeup acknowledgement packet to the sender.

$$
P_{\text {avg-wk }}=\frac{\left(P_{r w b n}+T_{w b n}\right)+\left(P_{t w a c k}+T_{t w a c k}\right)}{T}
$$

The BN transmits the data frame and receives Beacon and Ack packets from the BNC. The average power consumption for transmitting a data packet is calculated using (27). Similarly, the average power consumption for receiving the beacon and Ack is calculated using (28).

$$
\begin{aligned}
& P_{\text {avg-tx }}=P_{t x} \times \frac{T_{\text {data }}}{T} \\
& P_{\text {avg-rx }}=P_{r x} \times \frac{\left(T_{b}+T_{a c k}\right)}{T}
\end{aligned}
$$

The overhearing power $\left(P_{o h}\right)$ is only for wakeup radio transmission. The $(N-1)$ BNs overhear the wakeup radio packet. The Total overhearing power can be calculated using (29).

$$
P_{o h}=P_{r w b n} \times \frac{T_{w b n}}{T} \times(N-1)
$$

Power consumed due to other overheads includes sleep power, transition power, turnaround power, and overhearing power due to wakeup radio. The average overhead power consumption in the proposed MAC is calculated using (30).

$$
P_{a v g-o v}=P_{s l} \times \frac{T_{s l}}{T}+P_{t r} \times \frac{T_{t r}}{T}+P_{t} \times \frac{2 T_{t}}{T}+P_{r w b n} \times \frac{T_{w b n}}{T} \times(N-1)
$$

The total sleep time $\left(T_{s l}\right)$ for the proposed MAC is calculated using (31).

$$
T_{s l}=T-\left(T_{t r}+T_{b}+2 T_{t}+T_{d a t a}+T_{a c k}\right)
$$

Now we will calculate the delay for the above discussed MACs. As the network structure is single hop with a star topology, we have not considered many of the common delays that are inherent in a sensor network (e.g., queuing delay). The total delay for proposed MAC $\left(D_{o d}\right)$, WiseMAC ( WiseMAC $)$ B-MAC $\left(D_{B-M A C}\right)$, and X-MAC $\left(D_{X-M A C}\right)$ can be expressed using (32), (33), (34), and (35), respectively.

$$
\begin{aligned}
& D_{o d}=T_{d a t a}+T_{a c k}+T_{b}+2 T_{t}+T_{t r}+T_{w b n}+T_{w a c k} \\
& D_{\text {WiseMAC }}=T_{\text {data }}+T_{a c k}+T_{t}+T_{t r}+T_{p x}+\frac{T_{w k}}{2} \\
& D_{B-M A C}=T_{\text {data }}+T_{a c k}+T_{p}+T_{t}+T_{t r}+T_{p x} \\
& \left.D_{X-M A C}=T_{\text {data }}+2 T_{a c k}+N_{\text {probe }} \times T_{p}+3 T_{t}+T_{t r}+T_{(7)}\right)
\end{aligned}
$$

\subsection{BAN network setup for simulations}

We have considered a star topology with $10 \mathrm{BNs}$ for simulation as in the case of analysis. They are randomly deployed in a flat plain. The example topology is shown in Figure 15. Node 0 acts as the BNC. Rest of the nodes (BNs) are one hop away from the BNC. To validate our 


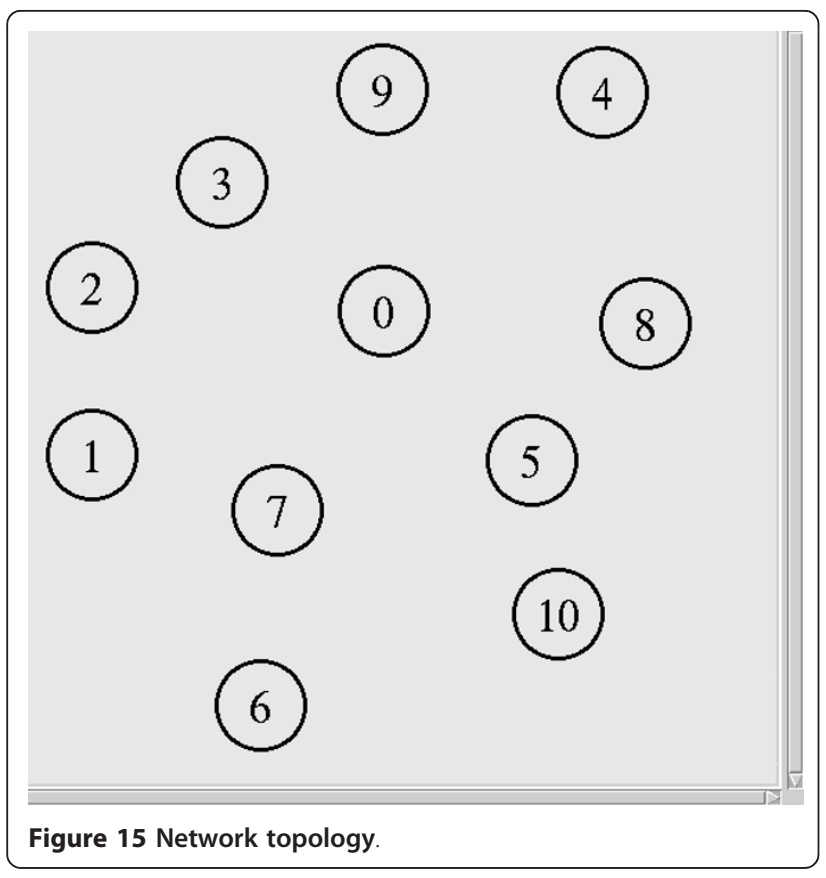

theoretical model, we simulated the works in Network Simulator NS-2 (release v2.31). We have used our own $\mathrm{C}++$ code. We have assumed ideal channel setup with no considerations for channel fading or other such behavior. The BNs are randomly placed in a 3-m area. The distance between a $\mathrm{BN}$ and $\mathrm{BNC}$ is between 0.1 and $3.0 \mathrm{~m}$. Each $\mathrm{BN}$ is assumed to have a wakeup radio transceiver. A BN can act and respond according to instructions from BNC. Wakeup table was generated for each $\mathrm{BN}$ using predefined $\lambda$ which we have taken from $[21,22]$. Data are generated based on the wakeup table for normal traffic and according to Poisson arrival for random (emergency) traffic. The packet sizes are taken as mentioned in Figure 10. For simulation, we have considered fixed data rate for all the BNs in the network. The power consumption is calculated for a BN for completion of a successful data transmission. We have also included the power consumption in the sleep state. The delay is calculated from the time a wakeup radio packet is sent until the data packet is transmitted and the Ack packet is received. We have taken the average of ten simulation runs.

\subsection{Results and discussion}

Traffic arrives at BNC with mean rate $\lambda_{e}$ for emergency traffic and $\lambda_{o}$ for normal/on-demand traffic. Authors of $[21,22]$ have provided some realistic values of arrival rate for various BNs. We have used these as reference and constructed the wakeup table with keeping in mind a real scenario for patient health monitoring. Arrival rate for emergency, $\lambda_{e}$ depends on the traffic condition
Table 7 Input parameters

\begin{tabular}{llllll}
\hline \multicolumn{5}{l}{ Common parameters } \\
\hline$P_{t x}$ & $27 \mathrm{~mW}$ & $P_{\text {active }}$ & $0.005 \mathrm{~mW}$ & $L_{\text {beacon }}$ & 10 Bytes \\
$P_{r x}$ & $1.8 \mathrm{~mW}$ & $R$ & $25 \mathrm{~kb} / \mathrm{s}$ & $L_{\text {ack }}$ & 10 Bytes \\
$T_{\text {data }}$ & $16 \mathrm{~ms}$ & $L_{\text {data }}$ & $50 \mathrm{Bytes}$ & $L_{\text {wup }}$ & 8 Bytes \\
$T_{\text {ack }}$ & $3.2 \mathrm{~ms}$ & $P_{\text {tr }}$ & $0.004 \mathrm{~mW}$ & $N$ & 10 \\
$T_{b}$ & $3.2 \mathrm{~ms}$ & $T_{\text {tr }}$ & $0.8 \mathrm{~ms}$ & $\theta$ & $30 \mathrm{ppm}$ \\
$P_{t}$ & $14.39 \mathrm{~mW}$ & $T_{\text {cca }}$ & $3 \mathrm{~ms}$ & & \\
$T_{t}$ & $0.4 \mathrm{~ms}$ & $P_{\text {sl }}$ & $0.004 \mathrm{~mW}$ & & \\
\hline
\end{tabular}

of the nodes and occurrence is rare compared to normal traffic. For simplicity, we have taken the average value for $\lambda_{e}=0.00005$. We have compared our model against B-MAC, X-MAC, WiseMAC, and ZigBeee. The input parameters for the simulation work mentioned in Tables 7 and 8 are taken from $[9-11,15,23]$.

Average power consumption and delay for the proposed MAC protocol are calculated. We have considered average power in the sleep, wakeup and communication state for each $\mathrm{BN}$ on a fixed time scale for packet inter-arrival time. Due to on-demand nature of the proposed protocol, it uses less power.

The comparison for average power consumption is shown in Figure 16. Use of wakeup radio improves performance of the proposed MAC. This is due to the basic fact that in our model, we do not have any duty cycle or need of periodic wakeup by a BN itself. Other MACs spend power in periodic channel assessment and polling activities. Reducing these overheads in the proposed MAC improves the overall performance. Overhearing is also a major cause of power wastage. Power is also wasted due to idle listening while waiting by the sender and receiver nodes in case of the other MACs. The proposed MAC completely removes the idle listening, thereby saving power. The effective use of wakeup table for periodic communications reduces power consumption of the BNs.

Figure 17 shows the delay comparison. It is found that the proposed MAC outperforms all other MACs. The delay remains fairly constant in our proposed model and is reasonable for delay sensitive application scenarios. The fact is that we used purely TDMA based mechanism and has only radio wakeup delay and transmission delay. This is evidently a major point to use

Table 8 Specific input parameters for each MAC

\begin{tabular}{llllll}
\hline Proposed & MAC & \multicolumn{2}{l}{ Wise MAC } & \multicolumn{2}{l}{ X-MAC } \\
\hline$P_{\text {twbn }}$ & $1.4 \mathrm{~mW}$ & $T_{w k}$ & $400 \mathrm{~ms}$ & $T_{w k r x}$ & $43.35 \mathrm{~ms}$ \\
$P_{\text {rwbn }}$ & $0.4 \mathrm{~mW}$ & $T_{p}$ & $20 \mathrm{~ms}$ & $T_{p}$ & $2.41 \mathrm{~ms}$ \\
$T_{\text {wbn }}$ & $2.56 \mathrm{~ms}$ & B-MAC & & $N_{\text {probe }}$ & $3.86 \mathrm{~ms}$ \\
$P_{\text {twack }}$ & $1.4 \mathrm{~mW}$ & $T_{w k}$ & $400 \mathrm{~ms}$ & & \\
$T_{w k}$ & $5.12 \mathrm{~ms}$ & $T_{p}$ & $86.7 \mathrm{~ms}$ & & \\
\hline
\end{tabular}




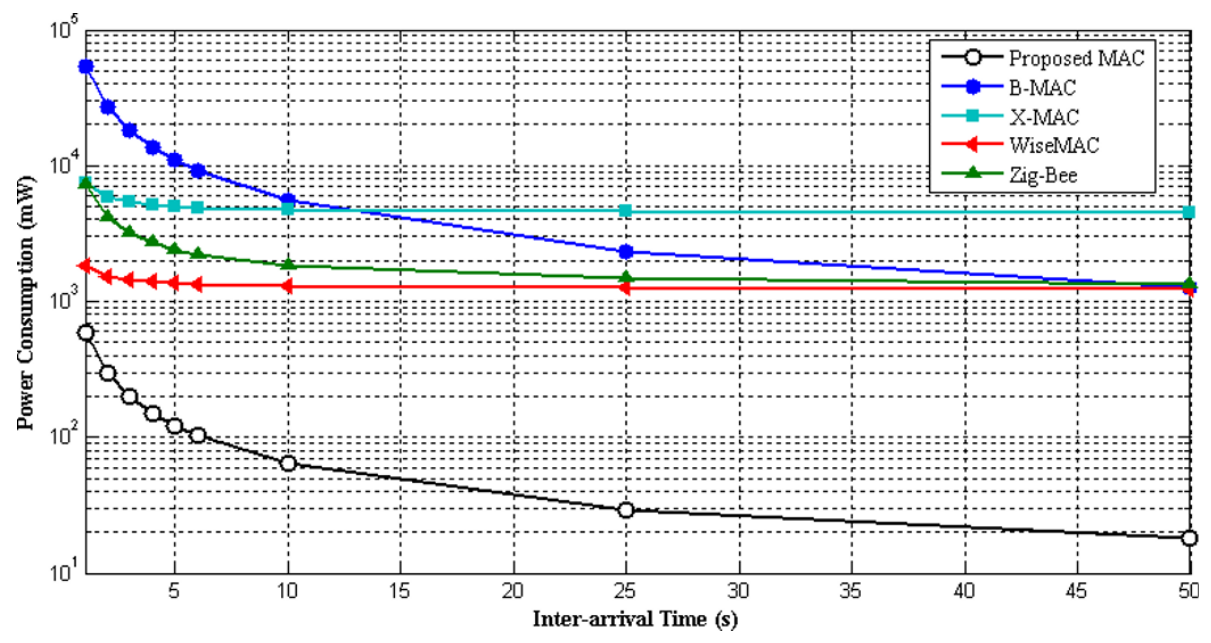

Figure 16 Power consumption.

wakeup radio-based system as it minimizes delay considerably in small single hop networks. In the case of B$\mathrm{MAC}$, it requires extra time to transfer packets from the source to the destination. This is because the entire preamble has to be sent, even though the receiver is already awake. The length of the preamble is also an issue in BMAC. X-MAC acquires delay as the packet inter-arrival time increases due to extra strobe preambles.

Wakeup interval significantly affects the power consumption of the duty cycle-based protocols. Protocols such as WiseMAC, ZigBee, and X-MAC need to adjust their wakeup period to optimize performance. Since our proposed method does not use duty cycle and periodic sampling or channel assessment, power consumption does not change as shown in Figure 18. Due to ondemand nature of the proposed protocol, wakeup intervals are controlled by BNC. This allows an ondemand scheme perform better than a variable duty cycle-based MACs.

The overheads in a MAC protocol play a major role in power consumption. Reducing the overheads is one of the major design goals of the proposed MAC. The proposed MAC tries to minimize the overheads through the use of wakeup table and on-demand communication. Every BN can reduce power consumption by switching off its main radio and thereby avoiding periodic wakeup and channel assessment. The default sleeping state for BNs further helps in reducing the power consumption due to overhearing neighbor BN's communication. The overhearing only happens at the wakeup radio in the proposed MAC for which the power consumption is very low. Figure 19 shows that the proposed

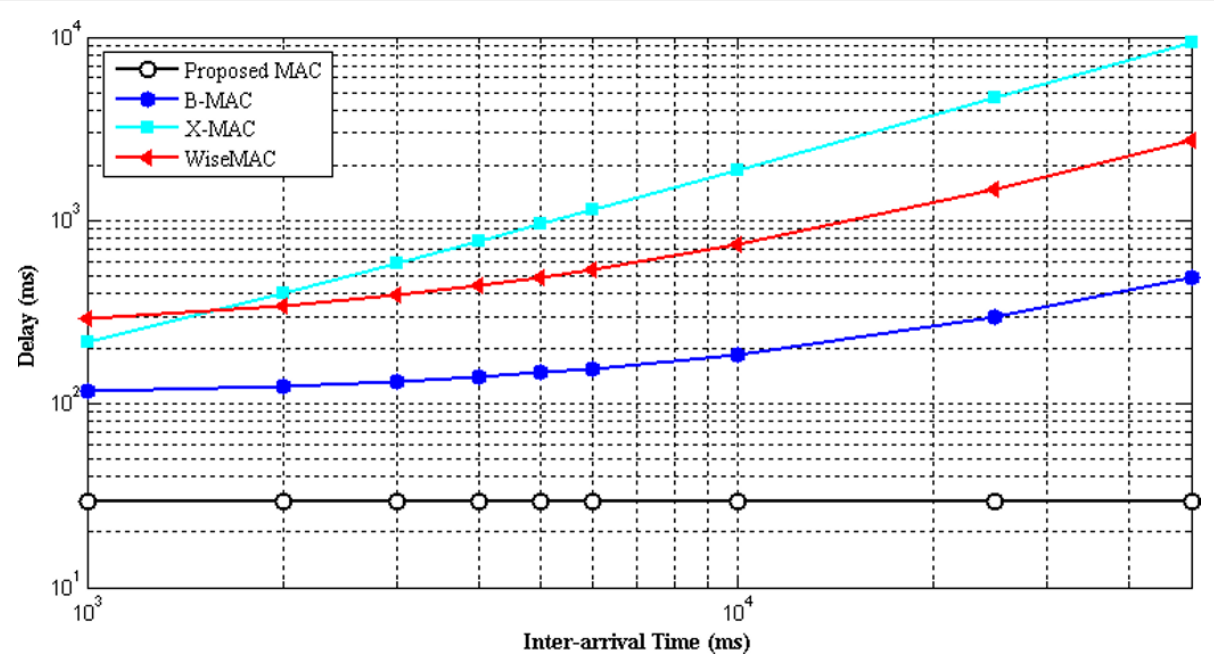

Figure 17 Delay. 


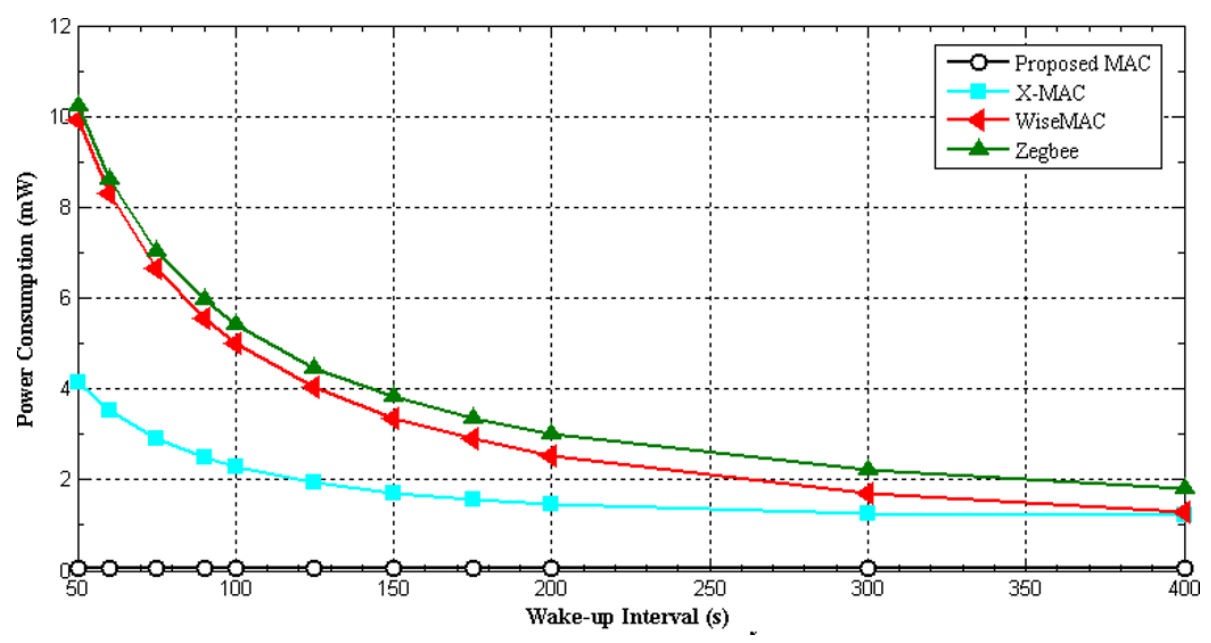

Figure 18 Wakeup interval versus power consumption.

MAC has done well to minimize the power consumption due to overheads.

Figure 20 shows the lifetime comparison. It can be seen that the lifetime for the proposed MAC has increased significantly. The initial big difference in the lifetime for low events/day is due to the fact that the proposed MAC is efficient in reducing potential causes of power waste by avoiding channel assessment, channel polling as employed by WiseMAC, ZigBee, B-MAC, and $\mathrm{X}$-MAC. In the case of low events per day, the proposed MAC has high lifetime due to maximized sleep time for BNs. This is achieved through the centrally managed wakeup table which is coordinated by the BNC. Rest of the BNs can simply go to sleep state when further data communication is not required. Traffic-based wakeup table with support from wakeup radio helps saving significant amount of power and thereby increase the lifetime of the BNs. As the activities increased in the network, the lifetime also decreases. The proposed MAC still outperforms other MACs when number events per day increases.

We have further validated our model by comparing the results of analysis with simulated data. The comparison of results shows similar trends as shown in Figure 21 . The small difference between theoretical and simulation curves is due to the randomness in the emergency traffics.

\section{Conclusions}

Application of sensor networks in healthcare is increasing fast. BAN is one of the major growth areas. In this article, we propose a power efficient MAC protocol for BAN using wakeup radio. The motivation of this study is that using a suitable out-of-band wakeup

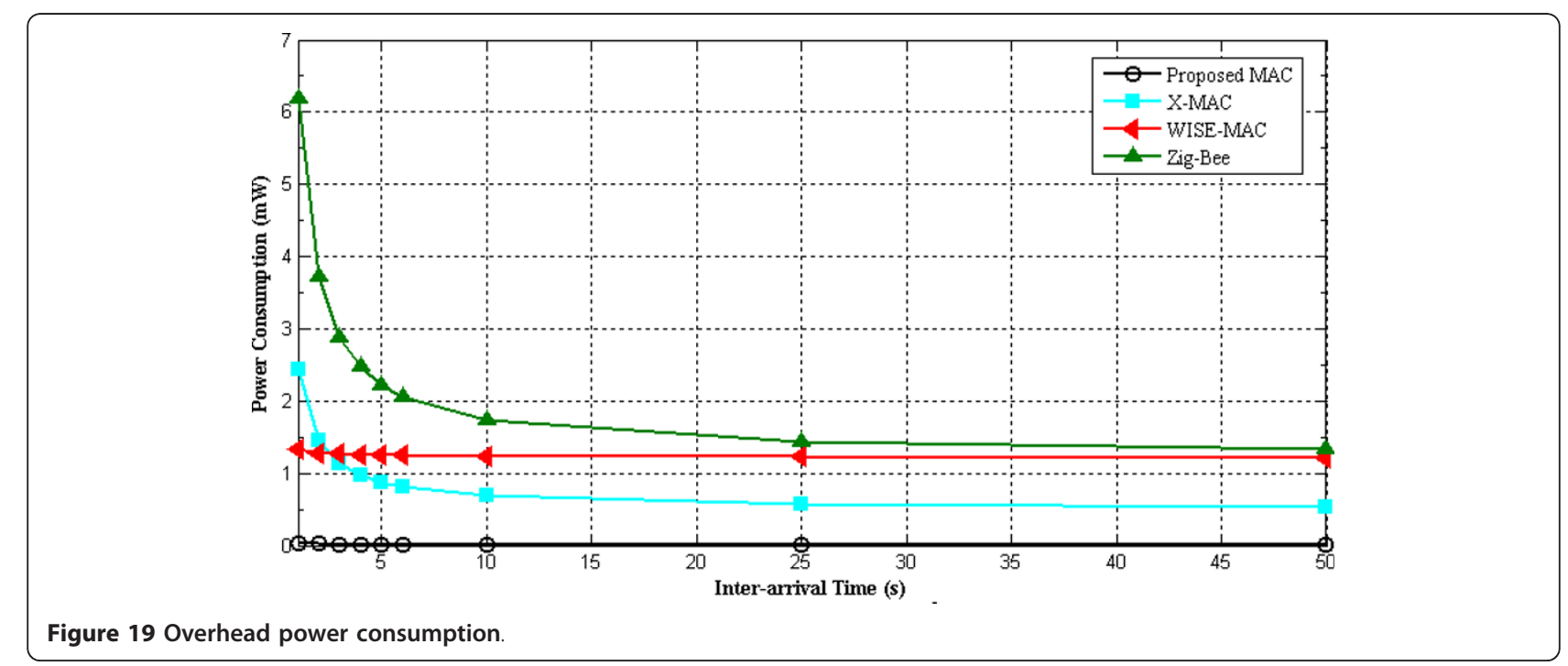




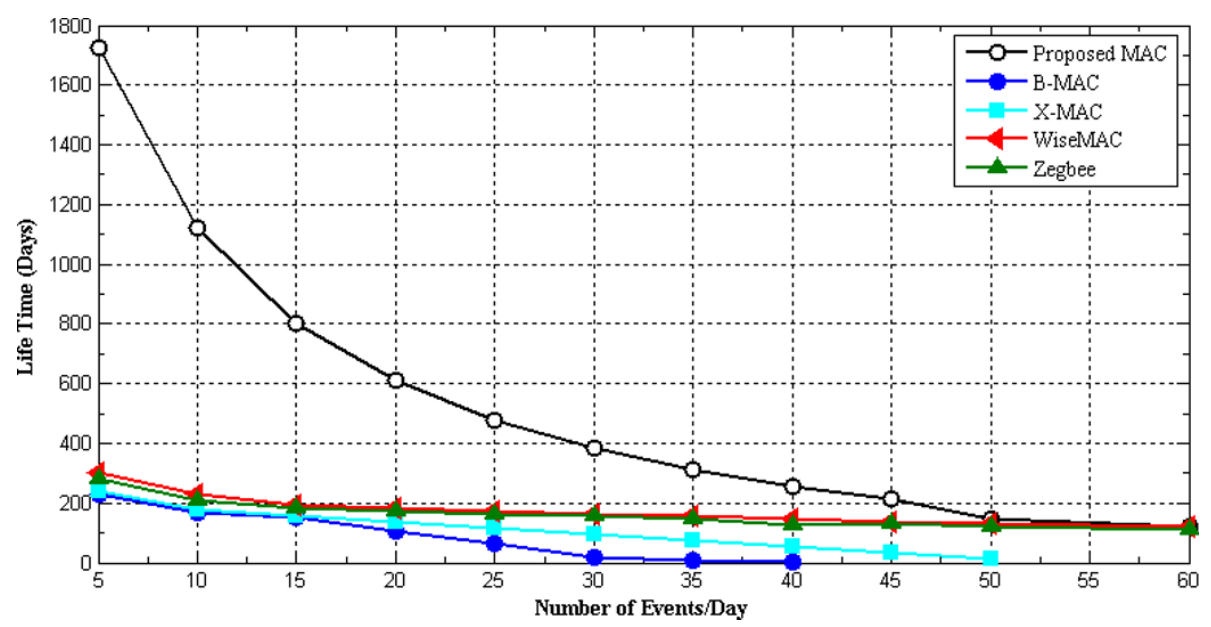

Figure 20 Lifetime.

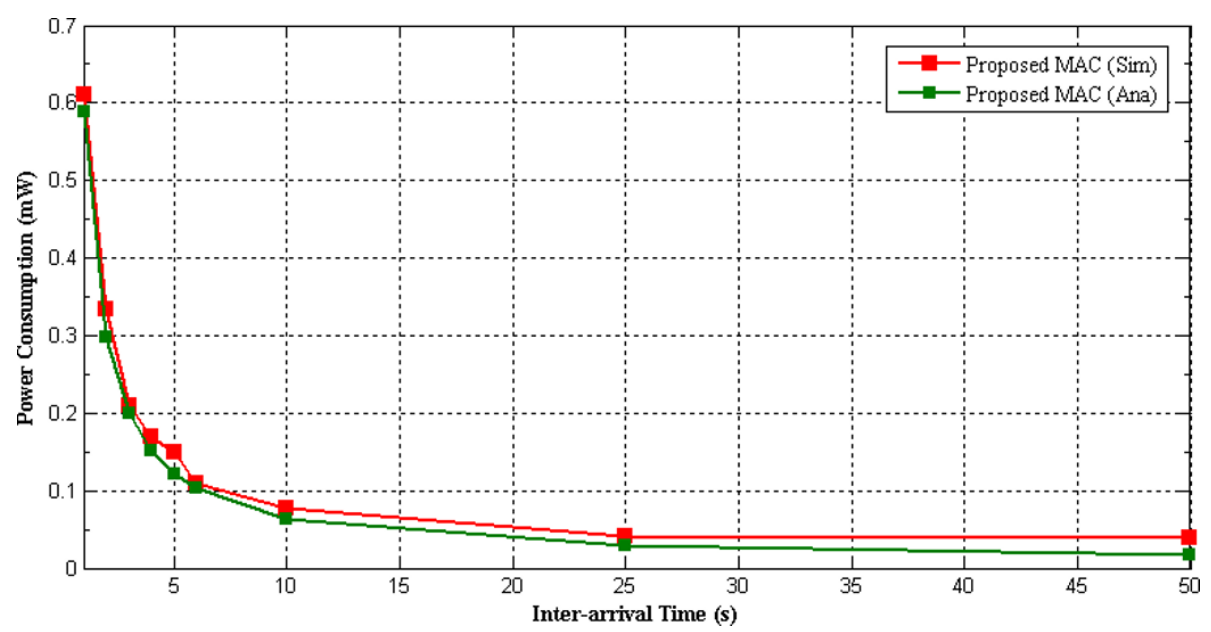

Figure 21 Simulation versus analysis comparison.

mechanism, a significant amount of power can be saved and thereby increase the lifetime. We discuss a model using wakeup radio. We covered periodic/normal communication as well as random/emergency communication. We have analyzed the power consumption and delay for the proposed protocol and then simulated it. Our method is found to be better in terms of power efficiency and delay. We have successfully demonstrated that a wakeup radio-based system can reduce overheads and enhance the lifetime. Future works include increasing number of neighbor nodes, security, QoS analysis and implement multi hop mechanisms in a real time test bed.

\section{Acknowledgements}

This research was supported by the National Research Foundation of Korea (NRF) grant funded by the Korea government (MEST) (No. 2010-0018116).

\section{Competing interests}

The authors declare that they have no competing interests.

Received: 6 April 2011 Accepted: 6 February 2012

Published: 6 February 2012

\section{References}

1. R MacRuairi, M Keane, G Coleman, A wireless sensor network application requirements taxonomy, in Proc Second International Conference on Sensor Technologies and Applications, SENSORCOMM '08, Cap Esterel, France, pp. 209-216 (2008)

2. IEEE 802.15.6, Application summary, 15-08-0406-00-0006-tg6-applicationsmatrix.xIs. (2008)

3. IEEE 802.15.6, Technical requirement documents (TRD), 15-08-0644-09-0006tg6-technical-requirements- document. (November 2008)

4. L Gu, J Stankovic, Radio-triggered wake-up capability for sensor networks, in Proceedings of the 10th IEEE Real-Time and Embedded Technology and Applications Symposium, RTAS 2004, Toronto, Canada, pp. 27-36 (May 2004)

5. R Falk, H-J Hof, Fighting insomnia: a secure wake-up scheme for wireless sensor networks, in Third International Conference on Emerging Security Information, Systems and Technologies, SECURWARE '09, Athens/Glyfada, Greece, pp. 191-196 (2009) 
6. N Pletcher, JM Rabaey, Ultra-low power wake-up receivers for wireless sensor networks. Ph.D. Dissertation, EECS Department, University of California, Berkeley (May 2008)

7. W Ye, J Heidemann, D Estrin, An energy-efficient MAC protocol for wireless sensor networks, in Proceedings Twenty-First Annual Joint Conference of the IEEE Computer and Communications Societies, INFOCOM 2002, vol. 3. New York, NY, USA, pp. 1567-1576 (2002)

8. T van Dam, K Langendoen, An adaptive energy-efficient MAC protocol for wireless sensor networks, in Proceedings of the First ACM Conference on Embedded Networked Sensor Systems, Los Angeles, CA, USA, pp. 171-180 (November 2003)

9. A El-Hoiydi, J-D Decotignie, WiseMAC: an ultra low power MAC protocol for the downlink of infrastructure wireless sensor networks, in Proceedings Ninth International Symposium on Computers and Communications, ISCC'04, vol. 1. Alexandria, EGYPT, pp. 244-251 (July 2004)

10. J Polastre, J Hill, D Culler, Versatile low power media access for wireless sensor networks, in Proceedings of the 2nd International Conference on Embedded Networked Sensor Systems, ser. SenSys '04, New York, NY, USA, ACM, pp. 95-107 (2004)

11. M Buettner, GV Yee, E Anderson, R Han, X-MAC: a short preamble MAC protocol for duty-cycled wireless sensor networks, in Proceedings of the 4th International Conference on Embedded Networked Sensor Systems, ser. SenSys '06, New York, NY, USA, ACM, pp. 307-320 (2006)

12. S Marinkovic, E Popovici, C Spagnol, S Faul, W Marnane, Energy-efficient Iow duty cycle MAC protocol for wireless body area networks. IEEE Trans Inf Technol Biomed. 13(6), 915-925 (2009)

13. M Miller, N Vaidya, A MAC protocol to reduce sensor network energy consumption using a wakeup radio. IEEE Trans Mobile Comput. 4(3), 228-242 (2005)

14. I Demirkol, C Ersoy, E Onur, Wake-up receivers for wireless sensor networks: benefits and challenges. IEEE Wirel Commun. 16(4), 88-96 (2009)

15. P Le-Huy, S Roy, Low-power $2.4 \mathrm{GHz}$ wake-up radio for wireless sensor networks, in IEEE International Conference on Wireless and Mobile Computing Networking and Communications, 2008. WIMOB '08, Avignon, France, pp. 13-18 (October 2008)

16. J Jung, $\mathrm{K} \mathrm{Ha}$, J Lee, Y Kim, D Kim, Wireless body area network in a ubiquitous healthcare system for physiological signal monitoring and health consulting. Int J Signal Process Pattern Recogn. 1, 47-54 (2008)

17. C Ding, $X W u, Z$ Lv, Design and implementation of the Zigbee-based body sensor network system, in 5th International Conference on Wireless Communications, Networking and Mobile Computing, WiCom '09, Beijing, China, pp. 1-4 (September 2009)

18. H Cao, X Liang, I Balasingham, VCM Leung, Performance analysis of ZigBee technology for wireless body area sensor networks, in Ad Hoc Networks, ser. Lecture Notes of the Institute for Computer Sciences, Social Informatics and Telecommunications Engineering, vol. 28. (Springer, Berlin, 2010), pp. 747-761. doi:10.1007/978-3-642-11723-7_51

19. G Fang, E Dutkiewicz, BodyMAC: energy efficient TDMA-based MAC protocol for wireless body area networks, in 9th International Symposium on Communications and Information Technology, ISCIT 2009, Incheon, Korea, pp. 1455-1459 (September 2009)

20. N Timmons, W Scanlon, An adaptive energy efficient MAC protocol for the medical body area network, in 1st International Conference on Wireless Communication, Vehicular Technology, Information Theory and Aerospace Electronic Systems Technology, Wireless VITAE 2009, Aalborg, Denmark, pp. 587-593 (May 2009)

21. JY Khan, MR Yuce, F Karami, Performance evaluation of a wireless body area sensor network for remote patient monitoring, in 30th Annual International Conference of the IEEE Engineering in Medicine and Biology Society, EMBS 2008, Vancouver, Canada, pp. 1266-1269 (August 2008)

22. HC Keong, MR Yuce, Analysis of a multi-access scheme and asynchronous transmit-only UWB for wireless body area networks, in 31st Annual International Conference of the IEEE Engineering in Medicine and Biology Society (EMBC'09), Minnesota, USA, pp. 6906-6909 (September 2009)

23. J Ansari, D Pankin, P Mhnen, Radio-triggered wake-ups with addressing capabilities for extremely low power sensor network applications. Int J Wirel Inf Netw. 16, 118-130 (2009). doi:10.1007/s10776-009-0100-6

24. BV d Doorn, W Kavelaars, K Langendoen, A prototype low cost wakeup radio for the $868 \mathrm{MHz}$ band. Int J Senor Netw. 5, 22-32 (2009). doi:10.1504/ IJSNET.2009.023313 doi:10.1186/1687-1499-2012-33

Cite this article as: Al Ameen et al:: A power efficient MAC protocol for wireless body area networks. EURASIP Journal on Wireless Communications and Networking 2012 2012:33.

\section{Submit your manuscript to a SpringerOpen ${ }^{\mathcal{O}}$ journal and benefit from:}

- Convenient online submission

- Rigorous peer review

- Immediate publication on acceptance

- Open access: articles freely available online

- High visibility within the field

- Retaining the copyright to your article

Submit your next manuscript at $\gg$ springeropen.com 